American Journal of Pharmaceutical Education 2020; 84 (4) Article 7739.

\title{
COMMENTARY
}

\section{Addressing Sexual and Gender Harassment in Pharmacy Education to Improve Provider Wellness and Patient Care}

\author{
Rebecca Schoen, PharmD, ${ }^{a}$ Amy Henneman, PharmD ${ }^{b}$ \\ ${ }^{a}$ Texas Tech University Health Sciences Center, Jerry H. Hodge School of Pharmacy, Dallas, Texas \\ ${ }^{\mathrm{b}}$ Palm Beach Atlantic University, West Palm Beach, Florida \\ Submitted June 13, 2019; accepted October 26, 2019; published April 2020.
}

\begin{abstract}
Research indicates widespread sexual harassment has not significantly decreased in health care over the last several decades. Attention focused on sexual harassment in the last few years has prompted a renewed conversation in health care about these complex issues and the unique challenges they present. Given the significant implications for individuals, organizations, and patient care, addressing harassment should be a priority. Pharmacy schools and supporting pharmacy organizations should proactively address sexual and gender harassment and provide education about this issue.
\end{abstract}

Keywords: education, female, sexism, sexual harassment

A national conversation centered around the \#MeToo movement that began in 2017 sparked a focus on how sexual harassment affects providing and receiving health care. ${ }^{1,2}$ Sexual harassment has broader definitions and consequences than is routinely recognized by the general public. A 2018 National Academies of Science, Engineering and Medicine report defines sexual harassment as sexual coercion, unwanted sexual attention, and gender harassment. ${ }^{3}$ Gender harassment or "verbal and nonverbal behaviors that convey hostility, objectification, exclusion, or second-class status about members of one gender" is the most frequent type of sexual harassment, but the impact is often minimized. The National Academies report indicates gender harassment that is severe or pervasive can cause the same negative professional and psychological outcomes as sexual coercion, and increases the likelihood that other forms of sexual harassment will occur. Unfortunately, the National Academies report and other publications indicate sexual harassment in the workplace has not significantly decreased over the last several decades and remains prevalent in both the medical and academic environments for faculty members, residents, and students. ${ }^{3-8}$ A literature search across medical disciplines within the last 25 years indicates sexual harassment is widespread throughout the health care disciplines, including among nurses, physicians, chiropractors, physical therapists, dentists, and pharmacists. ${ }^{7,9-12}$ Many

Corresponding Author: Rebecca Schoen, Duquesne

University School of Pharmacy, Division of Pharmacy

Practice, 600 Forbes Ave., Pittsburgh, PA 15282. Tel: 412-

396-2367. Email: schoenr@duq.edu learners may experience these scenarios as students or interns before graduating in the health profession fields. ${ }^{8,13,14}$ Given the risks of sexual harassment facing health care providers across the disciplines, what are we as a pharmacy profession doing to prepare our students and residents to not only manage their learning and work environments safely, but to be an agent of change in this environment?

Broadening the conversation of who is impacted by sexual harassment is essential when understanding the scope and impact of this issue. Sexual harassment is often thought of as primarily a female issue, but it is not limited to those identifying as female. Some literature indicates women are more likely to feel threatened, with stronger negative emotions stemming from the interaction. ${ }^{3,15}$ There is also evidence that women tend to report rates at a higher prevalence, but reporting rates can be difficult to capture and men do report experiences of sexual and gender harassment. Approximately $16 \%$ of complaints filed with the US Equal Employment Opportunity Commission are from individuals identifying as male. ${ }^{16}$ In a study of pharmacists by Broedel-Zaugg and colleagues, female respondents experienced significantly more gender harassment and unwanted sexual attention than men. ${ }^{11}$ However, there were no differences between male and female respondents in the total number of occurrences of sexual coercion. Among nurse respondents of a survey in the United Kingdom, approximately one in 20 nurses cited being sexually harassed within the last three years and more male $(7 \%)$ than female $(5 \%)$ nurses experienced the harassment. ${ }^{17}$ Ultimately, the data do not suggest this is a female-only issue. Beyond sex and gender, overlapping cultures and identities can impact the types and 


\section{American Journal of Pharmaceutical Education 2020; 84 (4) Article 7739.}

severity of harassment experienced. Sexual or gender minorities are vulnerable to harassment at higher rates. ${ }^{3}$ Women of color often experience harassment differently and with more severe consequences. People with a disability or individuals who have migrated or immigrated to the United States are more likely to face harassment and additive levels of discrimination. Unfortunately, data on these populations in health care is limited.

In addition to the national dialogue about sexual harassment, a renewed professional focus on wellbeing and resilience is occurring within health care. ${ }^{18,19} \mathrm{On}$ an individual and organizational level, addressing wellness is critical, and we believe wellness includes addressing sexual harassment. Increasing harassment frequency correlates to worse job-related and psychological outcomes, but even occasional episodes of sexual harassment can lead to negative consequences. ${ }^{3}$ For medical trainees, frequent harassment is correlated with learners who are less likely to complete assignments and provide optimal care; have more emotional health problems, social and family disruptions, depression, anxiety, insomnia, and appetite loss; and are more likely to use alcohol to escape problems. ${ }^{8}$ Sexual harassment occurrence has one of the strongest relationships to female well-being relative to other job stressors. ${ }^{3}$ When sexual harassment occurs in the workplace, victims may start distancing themselves mentally or physically from their workplace, experience decreases in job satisfaction and commitment to their role, have increases in stress or decreases in productivity, or leave their role altogether. Considering the potential for loss of talent, employees withdrawing from the workplace, and increased employee stress, the ramifications of sexual harassment extend to larger organizations and our profession.

Addressing sexual harassment in health care could also impact the delivery of patient care. Research indicates the distinct roles of pharmacists and other health care providers place them in situations that may leave them vulnerable to experiencing sexual harassment, including during patient care. ${ }^{3}$ Health care professionals often work physically and emotionally close with patients and caregivers, forming a relationship based on trust and mutual respect. However, for the patient, these situations may create an illusion of intimacy. The relationship can be further complicated by the patient's disease, in particular those suffering from conditions such as mental illness, dementia, or behavioral disturbances. Based on the limited studies available regarding pharmacists by Fjortfot and colleagues and Broedel-Zaugg and colleagues, approximately $50 \%$ of female pharmacist survey respondents noted some form of sexual harassment during their career, with almost equal numbers of incidents involving patients, providers, and colleagues. ${ }^{10,11}$ Approximately $17 \%$ of physicians in a 2019 survey in the United Kingdom noted harassment originating from patients. ${ }^{17}$ The unique dynamic of the provider-patient relationship and ineffective coping strategies commonly reported by providers indicate that sexual harassment is a complex problem that health care providers can struggle to effectively resolve or manage. Sexual harassment is primarily driven by attempts to remove a power imbalance or address a violation of gender norms rather than attraction, so the inherent power imbalance between patient and provider can leave providers vulnerable., , $^{30,11,20}$ When caring for offenders, many female providers report employing strategies of "coldness" to patients, shortened visits, and avoiding open-ended questions and certain examinations to mitigate their risk. ${ }^{14}$ These strategies could negatively impact patient care and may increase the power imbalance felt by the patient and, therefore, the risk for harassment to the provider. These employed strategies seem at best ineffective and hint at a lack of training, available resources, and organizational support. Though objective data are lacking on this topic, the combination of these risk management strategies and the decline in provider wellness seem likely to worsen patient care outcomes. ${ }^{17}$

While sexual harassment can occur regardless of role, evidence suggests our learners are particularly vulnerable to mistreatment from patients and the health care team. Studies by Wolf and colleagues and Schulte and colleagues indicate that up to $81 \%$ of female medical students reported sexual harassment specifically from patients. $^{21,22}$ To address these behaviors, younger individuals with less practice experience are more likely to utilize indirect strategies such as joking or ignoring behaviors, which are often ineffective. ${ }^{12}$ Coworkers, supervisors, and preceptors can also be involved in these situations as consultants and attendings are commonly cited as sources of harassment for trainees in a hierarchical envionrment. ${ }^{3,8,13,23}$ In general, underreporting of sexual harassment is common as people confronted with harassment often ignore or appease the harasser rather than report because of fear of retaliation or negative outcomes. ${ }^{3}$ Learners are particularly unlikely to bring forward concerns regarding sexual and gender harassment despite evidence that they encounter sexual harassment more frequently. ${ }^{23}$ When a learner experiences or witnesses sexual harassment, the response of the superior in the room is critical. Unfortunately, trainees who report these situations are frequently ignored by their superiors and often the response is silence. ${ }^{4}$ We should not expect our students to be the leaders on this issue. The Academy must be proactive in advocating, discussing, 


\section{American Journal of Pharmaceutical Education 2020; 84 (4) Article 7739.}

and creating a culture in health care that addresses sexual harassment for our learners.

In alignment with the National Academies report, we suggest that our professional organizations have a responsibility to address the climate of our profession, schools, and health systems. ${ }^{3}$ Pharmacist participants in the study by Fjortoft and colleagues suggested a need for better training and education on how to manage sexual harassment prior to entering pharmacy practice. Additionally, they highlighted a need for education on how to deliver pharmaceutical care while also managing the barriers sexual harassment creates when initiated by the patient. The study also noted looking to pharmacy role models to demonstrate how to let the offender know in a professional manner that this behavior is not acceptable. These changes will require training students, residents, and superiors in how to manage such situations and modeling of proactive behaviors. As pharmacy educators, we have a unique opportunity to directly impact these identified needs.

The Academy should consider making changes to current educational strategies to address these concerns. The National Academies report details several recommendations for academic institutions, including training to develop skills for interrupting and intervening when harassment occurs, focusing on changing an individual's behavior rather than beliefs, clearly communicating behavioral expectations, specifying consequences for harassment behaviors, and providing education that extends beyond the avoidance of legal liability. ${ }^{3}$ While addressing sexual harassment at organizations and universities from a legal standpoint remains important, prevalence rates indicate that most policies and trainings are currently insufficient to impact sexual harassment rates. Freyd and colleagues suggest that the current training mindset has left many in society unaware of the full scope of consequences and dynamics involved in sexual harassment. ${ }^{24}$ They recommend moving away from a training only mindset, which implies reaching a fixed endpoint, and moving towards an education mindset. An education mindset implies the learner must acquire knowledge and understanding, which differs greatly from a mindset of compliance and rule following associated with training. An education mindset could explore how an individual's implicit bias may lead to actions contradictory to one's conscious belief and how society's understanding of sexual harassment evolves. For example, the percentage of the United States population that classifies various offending behaviors as harassment has grown over the last 25 years. ${ }^{3}$ A continuing education approach would allow for ongoing assessment and critical thinking as perspectives change and the body of research grows.
Specific educational strategies and recommendations to consider in the literature include discussions or role-playing scenarios to address boundary setting, allowing participants the opportunity to consider how to approach a challenging encounter and process the emotions stemming from these awkward and uncomfortable situations. ${ }^{4,5,23,25}$ If thoughtfully incorporated, these scenarios could provide a safe place for trainees to identify the behavior, reflect on how to appropriately manage the situation, use avoidance behaviors that could be employed without impacting patient care, and set the expectation that we are a team and advocate for one another. ${ }^{3}$ Teaching students and residents to use key phrases that both acknowledge and redirect the negative behavior can set clear, appropriate boundaries with patients. ${ }^{5}$ Increased education on boundary setting and teaching students that harassment of any kind is unacceptable could have broad impacts for additional dimensions of an individual's culture and identity as harassment can occur from causes other than a person's sex or gender.

In the practice setting, pharmacy students can learn and see effective policies, interactions, and advocacy in real-time if modeled by their preceptors. Recent medical literature calls for providers to address the situation in real time in order to challenge the status quo, while actively teaching and modeling an expectation for a respectful, safe work environment. ${ }^{4-6}$ If practitioners model ignoring the behavior or joking with the patient, students learn strategies that are unlikely to be successful if faced with a similar situation in the future. ${ }^{12}$ Open dialogue as well as visual reminders concerning policies prohibiting harassment can be helpful reminders to both patients and employees. Viglianti and colleagues propose a decision tree algorithm for physicians based on how safe the provider feels during the encounter with the patient, empowering the provider to take appropriate steps to stop the harassment from occurring. ${ }^{6}$ Clear policies documenting appropriate boundaries with attendings, team members, and patients should be in place. ${ }^{23}$ In an era of team based care, interprofessional sessions regarding expectations of conduct in the workplace from both members of the team as well as patients and their family members may help to influence workplace culture and begin to challenge the status quo. Encouraging advocacy and engaging in leadership at an organizational level could lead to structures and policies for reporting and preventing harassment. ${ }^{1}$

If we fail to address all aspects of harassment, we fail to address a critical component of wellbeing, to maximize the potential of pharmacists, and to prepare practice-ready students for what is a common experience in the health professions, but does not have to be considered an inevitable aspect of these jobs. If the issue of sexual 


\section{American Journal of Pharmaceutical Education 2020; 84 (4) Article 7739.}

harassment is ignored, it sends a message of acceptance, which can be harmful to our learners, patients, and profession. The dynamics of interprofessional teams, training environments or provider-patient relationships are challenging to navigate without additional concerns for harassment. We should not expect our learners to speak up or be prepared to handle these situations now or in future practice without further education. If we do not teach students how to address these incidents and advocate for themselves, the risk of our students not meeting their full potential and harm to their well-being persists. These implications extend to our schools, our organizations, and ultimately our patients. If we start to emphasize education that creates a safe working environment, these interventions could have an overlapping impact on other dimensions of identity as well. We should advocate for our learners in an issue that cuts across health care disciplines by modeling proactive behavior and discussing strategies to insist our students' educational experience and future career in pharmacy is free from harassment of any form.

\section{ACKNOWLEDGMENTS}

The authors would like to thank Amie Blaszczyk, PharmD, BCGP, BCPS, FASCP, for reviewing this commentary and for her feedback prior to submission.

\section{REFERENCES}

1. Choo EK, Byington CL, Johnson NL, Jagsi R. From \#MeToo to \#TimesUp in health care: can a culture of accountability end inequity and harassment? Lancet. 2019;393:499-502.

2. Freischlag JA, Faria P. It is time for women (and men) to be brave: a consequence of the \#MeToo movement. JAMA.

2018;319:1761-1762.

3. Johnson PA, Widnall SE, Benya FF, National Academies of Sciences Engineering and Medicine (U.S.). Committee on the Impacts of Sexual Harassment in Academia. National Academies of Sciences Engineering and Medicine (U.S.). Committee on Women in Science Engineering and Medicine, National Academies of Sciences Engineering and Medicine (U.S.). Policy and Global Affairs. Sexual harassment of women: climate, culture, and consequences in academic sciences, engineering, and medicine. Washington, DC: The National Academies Press; 2018.

4. Killeen OJ, Bridges L. Solving the silence. JAMA.

2018;320:1979-1980.

5. Cowan AN. Inappropriate behavior by patients and their familiescall it out. JAMA Intern Med. 2018;178:1441.

6. Viglianti EM, Oliverio AL, Meeks LM. Sexual harassment and abuse: when the patient is the perpetrator. Lancet. 2018;392:368-370. 7. Gleberzon B, Statz R, Pym M. Sexual harassment of female chiropractors by their patients: a pilot survey of faculty at the
Canadian Memorial Chiropractic College. J Can Chiropr Assoc. 2015;59:111-121.

8. Fnais N, Soobiah C, Chen MH, et al. Harassment and discrimination in medical training: a systematic review and metaanalysis. Acad Med. 2014;89:817-827.

9. Ferns T, Meerabeau L. Verbal abuse experienced by nursing students. J Adv Nurs 2008;61:436-444.

10. Fjortoft N, Han N, Lee M. Sexual harassment in the pharmacy: recent findings and implications for practice and education. Am J Pharm Educ. 1996;60:30-36.

11. Broedel-Zaugg K, Shaffer V, Mawer M, Sullivan DL. Frequency and severity of sexual harassment in pharmacy practice in Ohio. $\mathrm{J} \mathrm{Am}$ Pharm Assoc (Wash). 1999;39:677-682.

12. Cambier Z, Boissonnault JS, Hetzel SJ, Plack MM. Physical therapist, physical therapist assistant, and student response to inappropriate patient sexual behavior: results of a national survey. Phys Ther. 2018;98:804-814.

13. Komaromy M, Bindman AB, Haber RJ, Sande MA. Sexual harassment in medical training. N Engl J Med. 1993;328:322-326.

14. Lenhart SA, Klein F, Falcao P, Phelan E, Smith K. Gender bias against and sexual harassment of AMWA members in Massachusetts. J Am Med Womens Assoc (1972). 1991;46:121-125.

15. Phillips S. Sexual harassment of female physicians by patients. What is to be done? Can Fam Physician. 1996;42:73-78.

16. U.S. Equal Employment Opportunity Commission. Charges Alleging Sex-Based Harassment FY 2010 - FY 2018. https://

www.eeoc.gov/eeoc/statistics/enforcement/

sexual_harassment_new.cfm. Accessed Accessed Oct 22019.

17. Sexual harassment of UK doctors: report 2019. 2019. https://

www.medscape.com/slideshow/uk-doctors-sexual-harassment-20196011895. Accessed April 16, 2020.

18. ASHP Statement on Commitment to Clinician Wellbeing and Resilience. https://nam.edu/wp-content/uploads/2017/11/AmericanSociety-of-Health-System-Pharmacists_Commitment-Statement.pdf. Accessed April 16, 2020.

19. Enhancing Well-Being and Resilience Among the Pharmacist Workforce: A National Consensus Conference. 2019. https:// www.pharmacist.com/sites/default/files/audience/

APhA_Well_Being_Resilience_Report_\%200719.pdf. Accessed April 16, 2020.

20. Phillips SP, Schneider MS. Sexual harassment of female doctors by patients. $N$ Engl J Med. 1993;329:1936-1939.

21. Wolf TM, Randall HM, von Almen K, Tynes LL. Perceived mistreatment and attitude change by graduating medical students: a retrospective study. Med Educ. 1991;25:182-190.

22. Schulte HM, Kay J. Medical students' perceptions of patientinitiated sexual behavior. Acad Med 1994;69:842-6.

23. Manning M. What if a resident or medical student is raped? Hospitals' and academic medical centers' title IX obligations. AMA J Ethics. 2018;20:3-9.

24. Freyd JJ, Smidt AM. So you want to address sexual harassment and assault in your organization? Training is not enough; Education is necessary. J Trauma Dissociation. 2019:1-6.

25. Walsh A. Our white coats are not armour. Protecting physicians in the doctor-patient relationship. Can Fam Physician. 2005;51:1604-5, 9-11. 